\title{
Coverage and Connectivity of Ad Hoc Networks in Presence of Channel Randomness
}

\author{
Daniele Miorandi \\ Create-Net \\ Via Solteri 38 \\ 38100 - Trento (Italy) \\ email: daniele.miorandidcreate-net.it
}

\author{
Eitan Altman \\ INRIA \\ 2004 Route des Lucioles \\ 06902 - Sophia Antipolis (France) \\ email: eitan.altmanesophia.inria.fr
}

\begin{abstract}
In this paper, we present an analytical procedure for the computation of the node isolation probability in an ad hoc network in the presence of channel randomness, with applications to shadowing and fading phenomena. Such a probability coincides with the complement of the coverage probability, given that nodes are distributed according to a Poisson point process. These results are used to obtain an estimate of the connectivity features for very dense networks. For the case of superimposed lognormal shadowing and Rayleigh fading, the connectivity improvements achievable by means of diversity schemes are investigated.

Index Terms-connectivity, coverage, ad hoc networks, noisy channels.
\end{abstract}

\section{INTRODUCTION}

One of the research fields which have gained more attention by the scientific community in the last few years is that of self-organizing largely deployed wireless networks, variously referred to as multihop, ad hoc or packet radio networks. Slightly aside, a growing interest is deferred to wireless sensor networks [1], which, while presenting peculiar features, possess at the same time many of the characteristics of ad hoc networks.

One of the issues of more concern, for such networks, is that of limiting achievable performance, in terms of capacity, connectivity and coverage [2], [3], [4], [5], [6], [7]. However, almost all the published works rely on a simplistic model of channel propagation, where the randomness inherently present in radio communications is not considered. To the best of the authors knowledge, only a few papers ([8], [9], [10], and [11] for the unidimensional case) deal with such phenomena.

In particular, our work has been inspired by that of Bettstetter et al. [9], who analise the connectivity of an ad hoc network in the presence of lognormal shadowing. Their analysis starts with a semi-analytical procedure for the computation of the node isolation probability, from which, under the assumption of dense networks, an estimate of the connection probability can be obtained. At first, we computed, using a different approach, similar to that used in [12] for different purposes, a closed-form expression for the node isolation probability in the presence of channel randomness (and, in particular, of shadowing and fading phenomena). Lately, we found out that results for the shadowing case had already been obtained by Orriss and Barton [13], who subsequently extended the analysis to the superposition of shadowing and fading phenomena [14], [15]. In this paper, we generalize their approach in order to account for a generic random channel, and show that the previously known results follow as special cases of our analysis.

We then show that, under our assumptions, the coverage probability, an important performance metric and design parameter in sensor networks, coincides with the complement of the node isolation probability. Further, under the assumption of very dense networks (to be formalized later), we provide an estimate of the probability that any finite portion of the network is connected. The possibility of exploiting the channel randomness by means of diversity schemes is also considered, showing that the network connectivity features may be enhanced with the adoption of multiple antennas or equivalent diversity schemes. It is also shown that no connectivity enhancements can be achieved by any random power transmission selection technique. The analytical results are compared with the outcomes of numerical simulations.

The paper is organized as follows: Sec. II presents the framework and reports the computation of the node isolation probability under the channel models mentioned above. The results are then used in Sec. III to get an estimate for the connection probability in very dense networks. The impact of two basic diversity schemes is investigated in Sec. IV. Sec. V concludes the paper indicating some directions for future research.

\section{Node Isolation Probability ANALysis}

\section{A. System Model}

Let $\mathcal{N}$ be a Poisson point process over the space $\mathbb{R}^{2}$ of intensity $\lambda^{1}$. The points of the process represent the location of the devices. Given a finite Borel subset $A \in \mathbb{R}^{2}$, the number of points of $\mathcal{N}$ in $A$, denoted by $\mathcal{N}(A)$, is a Poisson random variable of intensity $\lambda \nu(A)$, where $\nu(A)$ is the Lebesgue measure of the set $A$. All devices transmit at a fixed power level $P_{t x}$, and a white noise of power $W$ is assumed to be present at the receiver.

In our study of connectivity, we neglect the impact of interference. Our results are thus valid in the presence of low traffic

\footnotetext{
${ }^{1}$ The assumption of a 2-dimensional space is not necessary in the analysis, which may be easily generalized to the generic space $\mathbb{R}^{d}, d \geq 2$.
} 
load or when an efficient MAC layer protocol is present, so that we may disregard the mutual interference generated by the devices. Although this may seem an optimistic assumption (the impact of interference on connectivity is widely discussed in [16], [17]), in our view the connectivity is a "limiting" performance, representing in some sense the supremum of the connectivity properties over the whole range of MAC protocols, and thus this assumption is not limiting.

The modeling of propagation in a radio channel is a complex task, which heavily depends on the environment under study (indoor, outdoor urban, outdoor land etc.). The received power is given by the product of $P_{t x}$ and the path loss $l$, which is usually described by means of random variables ${ }^{2}$. The mean of the path loss is given by a law of the type $K r^{-\alpha}$, where $K$ is a constant which depends on some physical layer system features and $r$ represents the transmitter-receiver distance ${ }^{3}$. The path loss exponent, $\alpha$, is usually in the range $(2,4)$, while for some particular application scenarios it can be taken as large as 10 [19].

As far as the randomness inherently present in the radio channel is concerned, two different effects are usually considered, which may be observed over different time (or space) scales. The first, referred to as shadowing, reflects the different propagation conditions which may be encountered by radio waves due to buildings, terrain roughness, foliage and other obstacles. In other words, it considers that two terminals, located at the same distance by a transmitter, may experience different received signal power. A widely acknowledged model for shadowing predicts the received power to be lognormally distributed (i.e. the received power, expressed in $\mathrm{dB}$, is normally distributed) around its mean [19]. The shadowing phenomena are also referred to as "large scale" or slow fading, to distinguish them from the "small scale" or fast fading phenomena. The latter terms describe one of the peculiarities of radio wave propagation, i.e. the rapid fluctuation of the amplitude of a radio signal over a short period of time or travel distance. (Since we are considering static networks, the fading has in our case to be understood as a phenomenon which acts over the spatial dimension.) In the presence of dense multipath conditions, each received signal may be modelled as the superposition of two orthogonal normal components, so that the signal envelope is distributed as a Rayleigh random variable. In turn, this leads to an exponential distribution for the received power [20].

While such a model, which accounts for lognormal shadowing and superimposed Rayleigh fading, is by no means omnicomprehensive, it is able to cover a wide range of environmental conditions, providing precious guidelines for network design and dimensioning.

\footnotetext{
${ }^{2}$ Note that, according to our definition, the path loss should be understood as "path gain".

${ }^{3}$ As widely discussed in [18], the fact that the path loss goes to $\infty$ when $d$ goes to zero may lead to optimistic conclusions in the analysis of scaling laws for dense networks. However, this phenomenon appears as long as we take interference into account. As stated therein, "For models neglecting interferences $(. .$.$) these changes from a strict power law attenuation function$ may be only second order effects on the performance of the network".
}

Our analysis starts with the computation of the probability of a node being isolated, denoted by $P_{I}$, meaning that none of the other nodes present in the network is able to communicate with it. We denote by $P_{S \mid \Gamma}(y)$ the packet success probability given that the received signal presents an average signal-tonoise ratio $(\mathrm{SNR}) \Gamma=y$. If good long codes are used, the function $P_{S \mid \Gamma}$ tends to approach a step function [12]: in such a case we will denote by $\Psi$ the SNR threshold which ensures the correct packet reception. (A similar model, called "physical model", has also been used in [3].) More generally, $P_{S \mid \Gamma}(y)$ may be used to account for the peculiarities of realistic modulation schemes or, as we will see, to add another degree of randomness. In this work, we assume that the fading processes on the various links are independent. While such assumption clearly does not reflect a real network environment (especially as the node density $\lambda$ becomes large), it still enables us to obtain some closed-form expressions which may be used as estimates for more realistic situations and compared with real testbeds. In particular, we could expect that, in the presence of correlated links, the connectivity properties of the network will degrade with respect to the independent case.

The analysis of coverage and connectivity in the presence of channel randomness could, in principle, be addressed using tools and results from stochastic geometry. In particular, our model could be thought of as a classical Boolean model [21], [22]. In that model, also known as Poisson grain model, to each point of a Poisson point process (referred to as "germ"), a random shape (called "grain") is associated. The various grains, which are usually assumed to be convex and isotropic, are independent and identically distributed. For example, we could consider circles with a fixed radius; this would correspond, in our framework, to a deterministic propagation law. However, when considering channel randomness, it turns out to be difficult to formally define and describe the resulting grain shapes. This prevents from a direct application of a stochastic geometry approach. In the following, we will however show that the formulas for the node isolation probability have a close resemblance to those of the fraction volume in a Boolean model; in such a way, we can consider an equivalent grain area which describes the effects of channel randomness.

In a sensor network framework, one interesting performance metric is the coverage probability, which plays a fundamental role in intrusion detection and other applications [23], [24], [25], [26], [27]. In particular, we focus on networks where sensing relies on wave propagation laws which are those which guide signal propagation in the ad hoc case. The node isolation probability is the probability that a typical node is not connected to any other nodes, i.e. that it is not covered by the "footprint" generated by all other nodes. On the other hand, the coverage probability $P_{c o v}$ is the probability that a typical point is covered. Since $\mathcal{N}$ is a Poisson process, we can exploit Slivnyak's theorem [21], obtaining:

$$
P_{\text {cov }}=1-P_{I} \text {. }
$$

In case $\mathcal{N}$ is a more general point process (stationary ergodic), the two quantities can be related by means of a Palm inversion 
formula [21].

Another interesting meaning of $P_{I}$ is that, in the limit $\lambda \rightarrow$ $+\infty, P_{I}$ converges to zero at the same rate at which the probability of having one infinite connected component in the network converges to one [28]. Note, however, that the arising of an infinite component does not imply network connectivity, the latter requiring stronger conditions.

\section{B. A Deterministic Path-Loss Model}

The deterministic case will be used, in the following, as a reference to show the impact of the channel randomness induced by the shadowing and fading phenomena. In the case of a deterministic channel model, there exist a deterministic distance $R$ (referred to, in the following, as the "communication range"), so that a node is able to communicate with all the nodes lying within distance $R$. The signal-to-noise ratio (SNR) in the presence of a transmitter-receiver distance of $r$ is given by:

$$
\gamma(r)=\frac{P_{t x} l(r)}{W},
$$

where in this case $l(r)=K r^{-\alpha}$. The communication range may be computed as the distance at which the SNR falls below the threshold $\Psi$. Thus, we get:

$$
R=\left(\frac{K P_{t x}}{W \Psi}\right)^{\frac{1}{\alpha}}
$$

This is indeed a classical Boolean model with deterministic (circular) shapes, and the node isolation probability is given by:

$$
P_{I}=e^{-\lambda \pi R^{2}}=e^{-\lambda \pi\left(\frac{K P_{t x}}{W \Psi}\right)^{\frac{2}{\alpha}}} .
$$

\section{The Impact of Channel Randomness}

Let us consider a case where the channel model presents a random component. We assume the path loss, given a transmitter-receiver distance $r$, to be described by a probability density function (pdf) $f_{l \mid r}(\cdot \mid \cdot)$, or, equivalently, by the corresponding cumulative distribution function $F_{l \mid r}(\cdot \mid \cdot)$. We denote by $l_{i}$ the loss on the path between the intended device and node $i$. A node is isolated if, for any $i$, the $\operatorname{SNR} \frac{P_{t x} l_{i}}{W}$ falls below the threshold $\Psi$.

We define a random variable $R$, which will be referred to as the communication range in the following, having cumulative distribution function:

$$
\begin{aligned}
F_{R}(a) & =P[\gamma(a) \leq \Psi]=P\left[l(a) \leq \frac{W \Psi}{P_{t x}}\right]= \\
& =P\left[l(r) \leq \frac{W \Psi}{P_{t x}} \mid r=a\right]=F_{l \mid r}\left(\frac{W \Psi}{P_{t x}} \mid a\right) .
\end{aligned}
$$

In the more general case where $P_{S \mid \Gamma}(\cdot)$ is not a step function, we have:

$$
F_{R}(a)=1-\int_{0}^{+\infty} f_{l \mid r}\left(\frac{W x}{P_{t x}} \mid a\right) P_{S \mid \Gamma}(x) \mathrm{d} x .
$$

Intuitively, the communication range determines the probability that two devices, located at distance $a$ from one another, are able to successfully communicate with each other. The deterministic path-loss model can be seen as a special case, having the whole mass concentrated on a single value $R$. We assume $0<E\left[R^{2}\right]<+\infty$. The cases $E\left[R^{2}\right]=0$ and $E\left[R^{2}\right]=+\infty$ are trivial and of no interest: in the first case our device is disconnected P-a.s., whereas in the second one it is connected with all other devices P-a.s. Triviality extends also to network connectivity (see [28] for a percolation argument). We now compute the distribution of the number of one-hop neighbors of a device. The derivation will thread the footprints of [13].

Let us denote by $P_{n}(r)$ the probability that there are $n$ nodes, within distance $r$ from the intended device, which can communicate in a single-hop fashion with it. The number of devices with which our node can communicate directly (referred to as one-hop neighbors in the following) follows the distribution law $P_{n}(+\infty)$.

The event $\{n$ one-hop neighbors are present within distance $(x+\delta x)\}$ can be written as the union of three disjoint events (up to the first order in $\delta x$ ):

1) there are $n$ one-hop neighbors within distance $x$ and no nodes are present in the anulus $\mathcal{C}(x, x+\delta x)$;

2) there are $(n-1)$ one-hop neighbors within distance $x$ and one device in $\mathcal{C}(x, x+\delta x)$ which can communicate with the intended node;

3 ) there are $n$ one-hop neighbors within distance $x$ and one device in $\mathcal{C}(x, x+\delta x)$ which cannot communicate with the intended node.

Then, following [13] we can write for $n \geq 1$ :

$$
\begin{aligned}
& \quad P_{n}(x+\delta x)=P_{n}(x)[1-2 \pi \lambda x \delta x]+ \\
& +P_{n-1}(x) 2 \pi \lambda x \delta x\left[1-F_{R}(x)\right]+P_{n}(x) 2 \pi \lambda x \delta x F_{R}(x)+o(\delta x) .
\end{aligned}
$$

For $n=0$, we have:

$P_{0}(x+\delta x)=P_{0}(x)[1-2 \pi \lambda x \delta x]+P_{0}(x) 2 \pi \lambda x \delta x F_{R}(x)+o(\delta x)$.

Taking $\delta x \rightarrow 0$, we get:

$$
\left\{\begin{array}{l}
\frac{\partial P_{n}(x)}{\partial x}=2 \pi \lambda x\left[1-F_{R}(x)\right]\left[P_{n-1}(x)-P_{n}(x)\right], \quad n \geq 1 ; \\
\frac{\partial P_{0}(x)}{\partial x}=-2 \pi \lambda x\left[1-F_{R}(x)\right] P_{0}(x) .
\end{array}\right.
$$

Now, let us define the generating function of $P_{n}(x)$ as:

$$
\Pi(x, z)=\sum_{n=0}^{+\infty} P_{n}(x) z^{n} .
$$

From (9) we get:

$$
\frac{\partial \Pi(x, z)}{\partial x}=2 \pi \lambda x\left[1-F_{R}(x)\right](z-1) \Pi(x, z) .
$$

Hence,

$$
\frac{\partial \ln \Pi(x, z)}{\partial x}=2 \pi \lambda x\left[1-F_{R}(x)\right](z-1) .
$$


Noting that $\Pi(0, z)=1$, and hence $\ln \Pi(0, z)=0$, we get, integrating (12) from 0 to $+\infty$ :

$$
\begin{array}{r}
\ln \Pi(+\infty, z)=(z-1) \pi \lambda \int_{0}^{+\infty} 2 x\left[1-F_{R}(x)\right] \mathrm{d} x= \\
=(z-1) \pi \lambda E\left[R^{2}\right] .
\end{array}
$$

Inverting, we find that the number of one-hop neighbors follows a Poisson distribution with intensity $\pi \lambda E\left[R^{2}\right]$. As a corollary, the node isolation probability is given by:

$$
P_{I}=P_{0}(+\infty)=e^{-\lambda \pi E\left[R^{2}\right]} .
$$

Note that (14) could be retrieved withour resorting to generating function, by simply integrating the second equation in (9). An alternative method for finding the relationship (14) is reported in the appendix.

The equation above predicts that our system behaves like a Boolean model with equivalent average area $\hat{S}=\pi E\left[R^{2}\right]$ [21]. Formula (14) is worth some comments. Indeed, it predicts that channel randomness may have a beneficial impact on network connectivity. This holds if and only if the physical phenomenon which induces the randomness leads to an increment in the second-order moment of the random variable $R$.

\section{Analysis in the Presence of Shadowing}

In [9] a semi-analytical approach is presented for the computation of the node isolation probability in a shadowing environment. In particular, the procedure is based on the distribution of the $k$-th nearest neighbor distances, but no closed-form formula is obtained. By following the procedure outlined in the previous subsection, we are able to get a closedform expression for $P_{I}$, from which it is possible to clearly see the impact of the different factors. Given a transmitter-receiver distance $\rho$, the pdf of the path loss is given by [12]:

$$
f_{l \mid r}(a \mid \rho)=\frac{1}{\sqrt{2 \pi} \sigma a} e^{-\frac{1}{2}\left(\frac{\ln a-\ln (K \rho-\alpha)}{\sigma}\right)^{2}},
$$

where $\sigma$, the lognormal spread, is the standard deviation of the Gaussian distribution describing the shadowing phenomenon. We then have:

$$
\begin{aligned}
E\left[R^{2}\right] & =\int_{0}^{+\infty} \mathrm{d} \rho 2 \rho\left[1-F_{l \mid r}\left(\frac{W \Psi}{P_{t x}} \mid \rho\right)\right]= \\
& =\int_{0}^{+\infty} \mathrm{d} \rho 2 \rho \int_{\frac{W \Psi}{P_{t x}}}^{+\infty} \mathrm{d} a \frac{1}{\sqrt{2 \pi} \sigma a} e^{-\frac{1}{2}\left(\frac{\ln a-\ln \left(K \rho^{-\alpha}\right)}{\sigma}\right)^{2}} .
\end{aligned}
$$

Substituting $x=\frac{\ln a-\ln K \rho^{-\alpha}}{\sigma}=\frac{\ln \left(\frac{a \rho^{\alpha}}{K}\right)}{\sigma}$, we get:

$$
E\left[R^{2}\right]=\int_{0}^{+\infty} \mathrm{d} \rho 2 \rho \int_{\frac{\ln \left(\frac{W \Psi \rho \alpha}{P_{t x} K}\right)}{\sigma}}^{+\infty} \mathrm{d} x \frac{1}{\sqrt{2 \pi}} e^{-\frac{x^{2}}{2}} .
$$

Since the integrals converge absolutely, we may apply Fubini's theorem, getting:

$$
\begin{gathered}
E\left[R^{2}\right]=\int_{-\infty}^{+\infty} \mathrm{d} x \int_{0}^{\left(\frac{e^{\sigma x} P_{t x} K}{\Psi W}\right)^{\frac{1}{\alpha}}} \mathrm{d} \rho 2 \rho \frac{1}{\sqrt{2 \pi}} e^{-\frac{x^{2}}{2}}= \\
=\int_{-\infty}^{+\infty} \mathrm{d} x\left(\frac{e^{\sigma x} P_{t x} K}{\Psi W}\right)^{\frac{2}{\alpha}} \frac{1}{\sqrt{2 \pi}} e^{-\frac{x^{2}}{2}}= \\
=\left(\frac{P_{t x} K}{\Psi W}\right)^{\frac{2}{\alpha}} \int_{-\infty}^{+\infty} \mathrm{d} x \frac{1}{\sqrt{2 \pi}} e^{-\frac{x^{2}}{2}+\frac{2 \sigma x}{\alpha}}=\left(\frac{P_{t x} K}{\Psi W}\right)^{\frac{2}{\alpha}} e^{\left(\frac{\sqrt{2} \sigma}{\alpha}\right)^{2}},
\end{gathered}
$$

where we used the following integral [29]:

$$
\int_{-\infty}^{+\infty} \mathrm{d} x \frac{1}{\sqrt{2 \pi}} e^{-\frac{x^{2}}{2}} e^{ \pm \beta x}=e^{\frac{\beta^{2}}{2}} .
$$

Hence the node isolation probability is given by:

$$
P_{I}=e^{-\lambda \pi\left(\frac{P_{t x} K}{\Psi W}\right)^{\frac{2}{\alpha}}} e^{\left(\frac{\sqrt{2} \sigma}{\alpha}\right)^{2}} .
$$

Please note that this result was implicitly derived in [13].

This suggests that the presence of lognormal shadowing improves the connectivity properties of the network, as predicted by the results in [10], [8]. Further, the node isolation probability is monotonically decreasing in the lognormal spread $\sigma$. This fact may also be seen as a byproduct of a more general result, which regards the possibility of finding a stochastic ordering ${ }^{4}$ among the distributions of the communication range with different values of the lognormal spread $\sigma$. Indeed, we have:

$$
\begin{aligned}
F_{R_{\sigma}}(a) & =1-\int_{0}^{\frac{\Psi W}{K P_{t x}}} \mathrm{~d} x \frac{1}{\sqrt{2 \pi} \sigma x} e^{-\frac{1}{2}\left(\frac{\ln \left(x a^{\alpha}\right)}{\sigma}\right)^{2}}= \\
& =\int_{-\infty}^{\frac{\ln \frac{\Psi W a^{\alpha}}{K P_{t x}}}{\sigma}} \frac{\mathrm{d} t}{\sqrt{2 \pi}} e^{-\frac{t^{2}}{2}}=\Phi\left(\frac{\ln \frac{\Psi W a^{\alpha}}{K P_{t x}}}{\sigma}\right)
\end{aligned}
$$

It is then easy to see that we may write:

$$
R_{\sigma_{1}} \preceq R_{\sigma_{2}}, \quad \sigma_{1} \leq \sigma_{2} .
$$

From [30], (22) is equivalent to

$$
E\left[f\left(R_{\sigma_{1}}\right)\right] \leq E\left[f\left(R_{\sigma_{2}}\right)\right], \quad \sigma_{1} \leq \sigma_{2}
$$

for any increasing function $f(\cdot)$. Hence, we have that:

$$
E\left[R_{\sigma_{1}}^{h}\right] \preceq E\left[R_{\sigma_{2}}^{h}\right], \quad \sigma_{1} \leq \sigma_{2}, h \geq 0 .
$$

Taking $h=2$ and recalling (14), we retrieve that the node isolation probability decreases monotonically with the lognormal spread $\sigma$.

In the limit $\sigma \rightarrow+\infty$ the behavior of the resulting network

\footnotetext{
${ }^{4}$ Given two random variables $A$ and $B$, we say that $A$ is stochastically smaller than $B, A \preceq B$ if $F_{A}(x) \geq F_{B}(x) \forall x \geq 0$ [30].
} 
will resemble that of a random graph [31], in that the spatial component will become negligible. In such limiting regime, the probability that a typical node is connected to any other node tends to $\frac{1}{2}$. Indeed the probability of connection to a node at distance $\rho$ is:

$$
1-F_{R_{\sigma}}(\rho)=Q\left(\frac{\ln \frac{W \Psi \rho^{\alpha}}{P_{t x} K}}{\sigma}\right),
$$

which for any finite $\rho$ tends to $Q(0)=\frac{1}{2}$ as $\sigma \rightarrow+\infty$. The probability that, given $n$ other nodes in the network, the typical node is isolated is $\frac{1}{2^{n}} \rightarrow 0$ as $n \rightarrow+\infty$. Since in our unbounded region there is P-a.s. an infinite number of nodes, our network will P-a.s. not present any isolated node, which provides an informal justification of the fact that $\lim _{\sigma \rightarrow+\infty} P_{I}=0$. On the other hand, if $\sigma \rightarrow 0$ we retrieve the deterministic path-loss model previously analyzed. It is interesting to note that in such limiting case, the connectivity properties are driven only by the features of the underlying spatial process; in this sense, we may regard the resulting network as a geometric random graph [32] (this relationship between ad hoc networks and geometric random graphs is not new, see [33]). In some sense, the partial randomness introduced by the lognormal shadowing may be expected to produce a network similar to a small world [34], [35], which is indeed known to present better connectivity properties than geometric random graphs.

Related results are obtained in [36], where the authors, by using a continuum percolation approach, show the beneficial impact of spread-out connections (i.e., a spread-out density for $R$ in our setting) on the network connectivity keeping a fixed $E\left[R^{2}\right]$. On the other hand, we demonstrated that some forms of channel randomness (e.g., lognormal shadowing) are able to improve connectivity features by actually enlarging $E\left[R^{2}\right]$ without any increase in $P_{t x}$. The interesting phenomenon observed in [36] impacts the critical density at which network percolation occurs. However, this is not directly related to the node isolation probability, but to its relationship with the probability of existence of an infinite component. Further, in the limit $\lambda \rightarrow+\infty$ the enhancement due to spread-out connections vanishes, since the probability of existence of an infinite component tends to 1 at the same rate as $P_{I}$ (which depends just on $E\left[R^{2}\right]$ ) tends to 0 [28].

It is worth remarking that these results do not account for the basic fact that a wireless channel, in reality, cannot amplify a signal, so that the impact of lognormal shadowing in a real setting is not completely clear.

In Fig. 1 we plotted some results, in terms of coverage probability versus the node density $\lambda$ for various values of the lognormal spread $\sigma$. The other system parameters are $\alpha=3.5$, $P_{t x}=1 \mathrm{mWatt}, K=10, W=0.01 \mathrm{mWatt}, \Psi=10 \mathrm{~dB}$. From the curves it is evident that lognormal shadowing may have a tremendous impact on network performance, leading to a large performance enhancement, with respect to the deterministic channel model (correspondent to $\sigma=0$ ), even for low values of the lognormal spread. In Fig. 2 we plotted the coverage probability versus the lognormal spread for various values of the path loss factor $\alpha$, with $P_{t x}=1$ mWatt, $K=10$, $W=0.01$ mWatt, $\Psi=10 \mathrm{~dB}, \lambda=0.01 \mathrm{~m}^{-2}$. The asymptote represents the coverage probability achieved with a deterministic channel model. Note that the path loss factor $\alpha$ plays a substantial role only for a narrow range of values for $\sigma$ (those corresponding to the critical transition).

In order to validate our results, we simulated a $100 \mathrm{~m} \times 100 \mathrm{~m}$ square, and took the same system parameters as above. We considered a node density $\lambda=0.04 \mathrm{~m}^{-2}$ and varied $\sigma$ between 0 (path loss only) and 3 . The corresponding communication graphs (in which two points are linked by an edge if and only if the corresponding devices are connected to each other) are plotted in Fig. 3, where it may be noticed the remarkable impact of even a small variation of the lognormal spread $\sigma$. We then computed the node isolation probability averaging over 1000 simulations. The results, for the case $\sigma=0$ and $\sigma=2$ are plotted in Fig. 4, where for the simulation results we reported the mean value. It may be seen that our analysis is able to closely follow the system behavior for a wide range of node density, our analysis slightly overestimating the node isolation probability. This effect is due to the fact that our analysis has been carried out for an unbounded region, neglecting thus the border effects that arise in the presence of a finite area [37]. In such a case, in fact, nodes placed close to the border regions are more likely to be isolated, since they can connect only to devices placed in a region of area smaller than $\pi E\left[R^{2}\right]$. The border effect, in our example, has an impact which is increasing with an increase in the mean covered area $\pi E\left[R^{2}\right]$, as it may be seen in Fig. 4.d. This explains the small mismatch between analysis and simulation data.

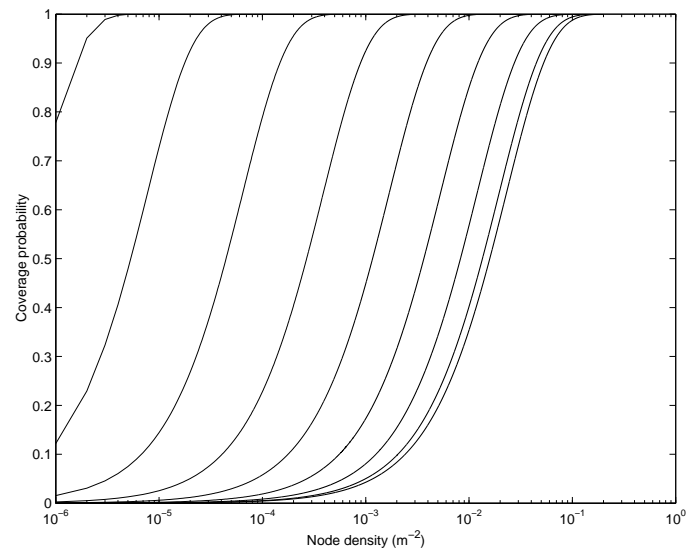

Fig. 1. Coverage probability vs. node density in the presence of lognormal shadowing, $\alpha=3.5, P_{t x}=1$ mWatt, $K=10, W=0.01$ mWatt, $\Psi=10$ $\mathrm{dB}, \sigma=0,1, \ldots, 10$ (a larger value of $\sigma$ corresponds to a curve to the left).

\section{E. Analysis in the Presence of Superimposed Lognormal Shad- owing and Rayleigh Fading}

The analytical procedure showed above may be easily extended to more complex scenarios, such as that where both lognormal shadowing and fast (Rayleigh) fading are simultaneously present [14]. Let $\Gamma$ be the average SNR 


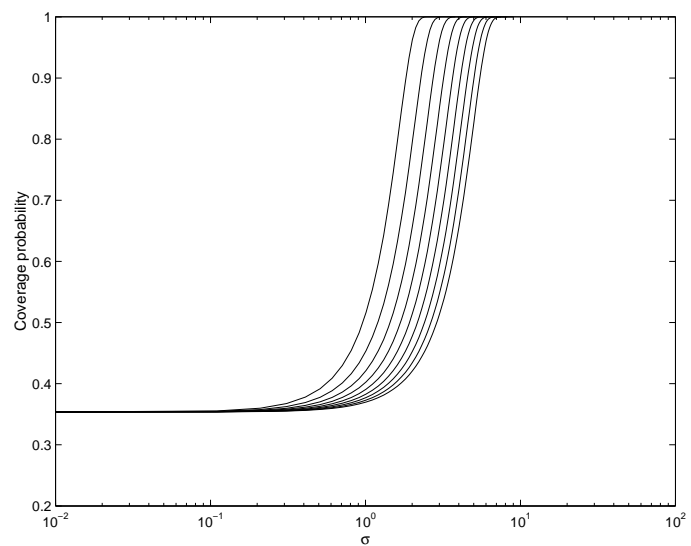

Fig. 2. Coverage probability vs. $\sigma$ in the presence of lognormal shadowing, $P_{t x}=1$ mWatt, $K=10, W=0.01$ mWatt, $\Psi=10 \mathrm{~dB}, \lambda=0.01 \mathrm{~m}^{-2}$, $\alpha=2,2.5, \ldots, 6$ (a larger value of $\alpha$ corresponds to a curve to the right).

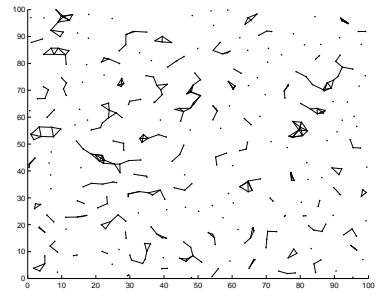

(a) $\sigma=0$

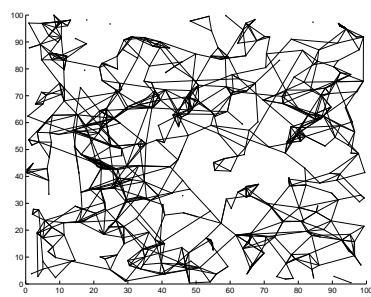

(c) $\sigma=2$

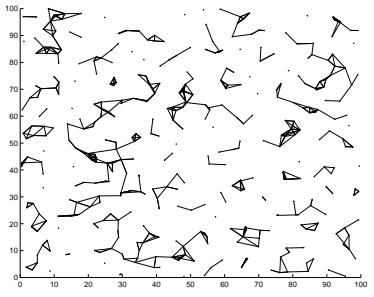

(b) $\sigma=1$

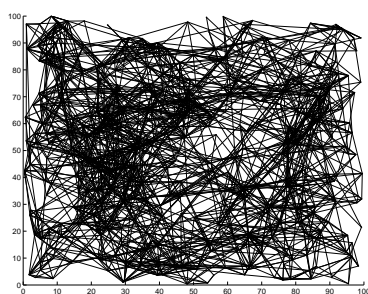

(d) $\sigma=3$

Fig. 3. Communication graphs for $\alpha=3.5, P_{t x}=1 \mathrm{mWatt}, K=10$, $W=0.01$ mWatt, $\Psi=10 \mathrm{~dB}, \lambda=0.04 m^{-2}$.

(meaning that it is averaged over the variations of the smallscale fading), and consider again a threshold-like success probability, with threshold equal to $\Psi$. The instantaneous SNR $\gamma$ is exponentially distributed with mean $\Gamma=y$ [20] so that we have:

$$
P_{S \mid \Gamma}(y)=\int_{\Psi}^{+\infty} \mathrm{d} a f_{\gamma \mid \Gamma}(a \mid y)=\int_{\Psi}^{+\infty} \mathrm{d} a \frac{1}{y} e^{-\frac{a}{y}}=e^{-\frac{\Psi}{y}} .
$$

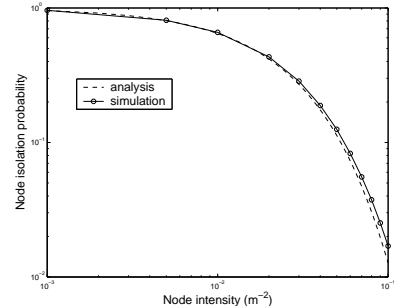

(a) $\sigma=0$

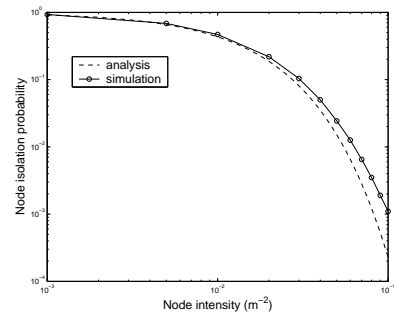

(c) $\sigma=2$

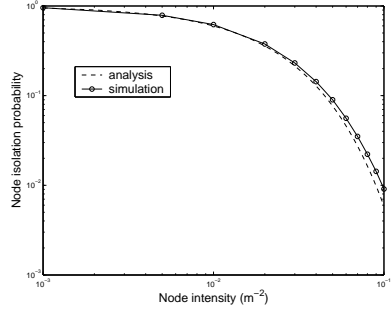

(b) $\sigma=1$

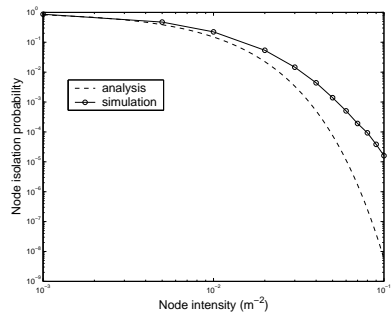

(d) $\sigma=3$
Fig. 4. Node isolation probability for $\alpha=3.5, P_{t x}=1 \mathrm{mWatt}, K=10$, $W=0.01$ mWatt, $\Psi=10 \mathrm{~dB}$.

Substituting in (14), we obtain:

$$
\begin{gathered}
P_{I}=e^{-\lambda \pi\left[\int_{0}^{+\infty} \mathrm{d} a \int_{0}^{+\infty} \mathrm{d} \rho P_{S \mid \Gamma}\left(\frac{a P_{t x}}{W}\right) 2 \rho f_{l \mid r}(a, \rho)\right]}= \\
=e^{-\lambda \pi\left[\int_{0}^{+\infty} \mathrm{d} a \int_{0}^{+\infty} \mathrm{d} \rho e^{-\frac{\Psi W}{a P_{t x}}} 2 \rho \frac{1}{\sqrt{2 \pi} \sigma a} e^{\left.-\frac{1}{2}\left(\frac{\ln a-\ln \left(K \rho^{-\alpha}\right)}{\sigma}\right)^{2}\right]}\right]}= \\
e^{-\lambda \pi\left(\int_{-\infty}^{+\infty} \mathrm{d} x \int_{0}^{+\infty} \mathrm{d} \rho \frac{1}{\sqrt{2 \pi}} e^{-\frac{x^{2}}{2}} 2 \rho e^{-\frac{\Psi W e^{-\sigma x} \rho^{\alpha}}{K P_{t x}}}\right)}
\end{gathered}
$$

From [29] we have:

$$
\int_{0}^{+\infty} \mathrm{d} \rho 2 \rho e^{-\frac{\Psi W e^{-\sigma x} \rho^{\alpha}}{K P_{t x}}}=\frac{2}{\alpha} \Gamma\left(\frac{2}{\alpha}\right)\left(\frac{\Psi W e^{-\sigma x}}{K P_{t x}}\right)^{-\frac{2}{\alpha}},
$$

where $\Gamma(\cdot)$ represents the usual Gamma function. Then, applying (28) and (19) to (27), we obtain:

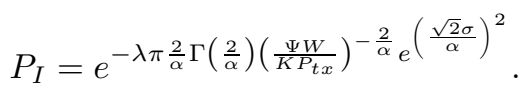

Since $y \Gamma(y) \leq 1$ for any $y \leq 1$, equality holding only for $y=1$, we have that, for $\alpha>2$ (note that the case $\alpha=2$ corresponds to free-space propagation, so that for any real system it is $\alpha>2$ ) Rayleigh fading reduces the connectivity properties of the network. This holds for any $\sigma$, so that the negative influence of the Rayleigh fading does not depend on the underlying shadowing phenomena.

In Fig. 5 a comparison between lognormal shadowing and superimposed shadowing and Rayleigh fading is shown. Notice that the Rayleigh fading, although having a negative impact 
on the connectivity properties of the network, does not change much the coverage probability.

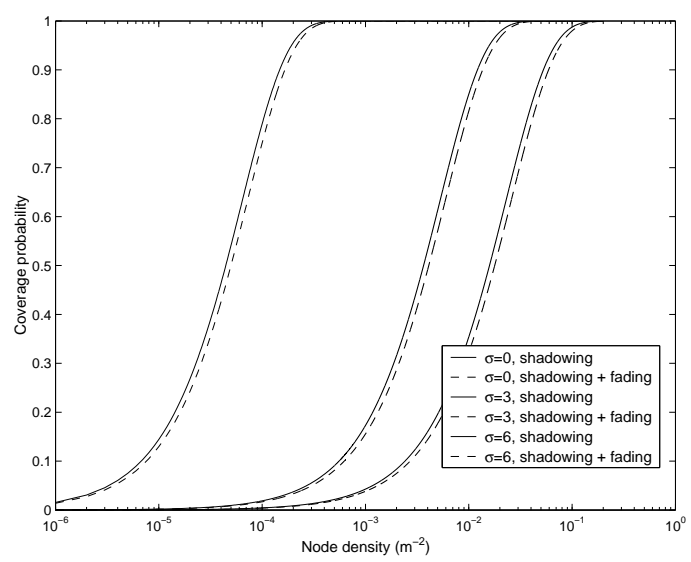

Fig. 5. Coverage probability vs. node density: the impact of Rayleigh fading; $P_{t x}=1$ mWatt, $K=10, W=0.01$ mWatt, $\Psi=10 \mathrm{~dB}, \alpha=3.5$, $\sigma=0,3,6$ (a larger value of $\sigma$ corresponds to a curve to the left).

\section{F. Random Transmission Power Selection}

The main result we can draw from (14) is that any mechanism able to increase the second-order moment of the transmission range enhances the network performance. Since the mean value of the transmission range is determined by physical layer constraints (noise, average transmission power, SNR threshold and path loss factor), it seems quite natural to look for a technique able to enhance the variance of the transmission range. In this view, the easiest thing to do would be to allow the terminals to independently randomly choose a power level $P_{t x}$ according to a distribution $F_{P_{t x}}(\cdot)$ on which we pose only a constraint on the mean value, namely:

$$
E\left[P_{t x}\right]=\int_{0}^{+\infty} \mathrm{d} a\left[1-F_{P_{t x}}(a)\right]=\omega .
$$

We get the following:

Proposition 1: Given an ad hoc network where nodes are distributed according to a Poisson point process of intensity $\lambda$, and in the presence of a deterministic channel model/lognormal shadowing/superimposed Rayleigh fading and lognormal shadowing, under the constraint (30), it is not possible to reduce the node isolation probability by means of any transmission power random selection scheme .

Proof: For the three channel models considered, we have:

$$
E\left[R^{2} \mid P_{t x}\right]=\xi\left(P_{t x}\right)^{\frac{2}{\alpha}},
$$

where $\xi=\left(\frac{K}{W \Psi}\right)^{\frac{2}{\alpha}}$ for the case of deterministic channel, $\xi=\left(\frac{K}{W \Psi}\right)^{\frac{2}{\alpha}} e^{\left(\frac{\sqrt{(2) \sigma}}{\alpha}\right)}$ for lognormal shadowing and $\xi=\frac{2}{\alpha} \Gamma\left(\frac{2}{\alpha}\right)\left(\frac{K}{W \Psi}\right)^{\frac{2}{\alpha}} e^{\left(\frac{\sqrt{(2) \sigma}}{\alpha}\right)}$ for superimposed lognormal shadowing and Rayleigh fading. Then, applying the total expectation theorem:

$$
E\left[R^{2}\right]=E\left[E\left[R^{2} \mid P_{t x}\right]\right]=\xi E\left[\left(P_{t x}\right)^{\frac{2}{\alpha}}\right] .
$$

For $\alpha \geq 2,(\cdot)^{\frac{2}{\alpha}}$ is a concave function. Then, applying Jensen's inequality, we have:

$$
E\left[R^{2}\right]=\xi E\left[P_{t x}^{\frac{2}{\alpha}}\right] \leq \xi E\left[P_{t x}\right]^{\frac{2}{\alpha}}=\xi \omega^{\frac{2}{\alpha}} .
$$

Since the r.h.s. of (33) is what we would get by taking $P_{t x}=$ $\omega$, we obtain that connectivity cannot be improved by means of any random selection scheme for the transmission power ${ }^{5}$

\section{Connectivity AnAlysis And Results}

\section{A. Asymptotic Connectivity}

One of the most important issues in ad hoc networks is that of connectivity, which can be posed in the following way: given a ball of radius $c$ (or any other finite convex subarea of the plane), what is the probability that the nodes therein forms a connected topology? In general, this question cannot be answered exactly (see [22] for more details). However, we may still get some insight when the network is "very dense", meaning that the intensity of our driving process $\mathcal{N}$ goes to infinity. This corresponds, roughly speaking, to a situation where the network is "not far" from connectivity, meaning that the probability of a node to be isolated is vanishing. In such a situation, the probability of the network being connected tends to the probability that no isolated nodes are present. This approximation, which clearly offers an optimistic bound on the probability of the network to be connected, has been shown in [9] to provide good results. Furthemore, such result is known to hold for the case of deterministic channel model [38], [32]. Consider balls of radius $\rho, D_{\rho}$. The number of points in $D_{\rho}$ forms a Poisson r.v. with intensity $\lambda \pi \rho^{2}$. Let us introduce the following approximations:

(i) the region $D_{\rho}$ is sufficiently large, so that border effects may be neglected [37];

(ii) the probability that a node is isolated, given that there are $n$ nodes in $D_{\rho}$, is given by $P_{I}$;

(iii) given $n$ nodes in $D_{\rho}$, the events $I_{k}=$ \{node $k$ is isolated $\}, k=1, \ldots, n$ are independent.

As a consequence, the probability of the event \{absence of isolated nodes in $D_{\rho} \mid n$ nodes are present $\}$ is given by $\left(1-P_{I}\right)^{n}$. Using the total probability theorem, we have:

$$
P[A]=\sum_{n=0}^{\infty} e^{-\lambda \pi \rho^{2}} \frac{\left[\lambda \pi \rho^{2}\left(1-P_{I}\right)\right]^{n}}{n !}=e^{-\lambda \pi \rho^{2} P_{I}} .
$$

where $A$ is the event $\{$ absence of isolated nodes $\}$. Notice that the approximations introduced above, while clearly representing a simplistic picture of the network behavior, have proven in [9] to provide a satisfactory level of approximation.

Denoting by $C$ the event $\{$ the network is connected $\}$, we have:

$$
\lim _{\lambda \longrightarrow \infty} P[A]-P[C]=0 .
$$

It is worth noting that such approximation is known to hold for the unidimensional case [39], [22].

\footnotetext{
${ }^{5}$ Note, however, that in a 3-dimensional network, if $\alpha<3$, random power selection schemes could indeed improve network connectivity.
} 
The node isolation probability $P_{I}$ is a function of $P_{t x}$; we shall allow $P_{t x}$ to be a function of $\lambda$ as well. Thus the dependence of $P[A]$ on $\lambda$ is through the behavior of $\lambda P_{I}$.

Using (35), we shall say that the network is asymptotically connected if $\lim _{\lambda \rightarrow+\infty} P[A]=1$.

Under the approximations $(i)-(i i i)$, from (34) a necessary and sufficient condition for asymptotic connectivity of any finite $D_{\rho}$ is:

$$
\lim _{\lambda \rightarrow \infty} \lambda P_{I}=0 .
$$

Let us assume that we are in the presence of a deterministic channel model/lognormal shadowing/superimposed Rayleigh fading and lognormal shadowing, and let the transmission power scale as:

$$
P_{t x}=\left(\frac{1}{\pi \xi} \cdot \frac{\ln \lambda+c(\lambda)}{\lambda}\right)^{\frac{\alpha}{2}},
$$

where the expression of $\xi$ depends on the channel model (see $\S$ II-F). For the three channel models considered, we have $P_{I}=$ $e^{-\lambda \pi \xi\left(P_{t x}\right)^{\frac{2}{\alpha}}}$. Using the expression (37), we have:

$$
P_{I}=e^{-(\ln \lambda+c(\lambda))}=\frac{e^{-c(\lambda)}}{\lambda} .
$$

From (36), a necessary and sufficient condition for asymptotic connectivity is $e^{-c(\lambda)} \rightarrow 0$, from which a necessary and sufficient condition for asymptotic connectivity is:

$$
\lim _{\lambda \longrightarrow \infty} c(\lambda)=+\infty \text {. }
$$

It is worth noting that the scaling law of the transmission power for asymptotic connectivity in dense networks is insensitive to the presence of shadowing and/or fading phenomena (up to a multiplicative factor). This insensitivity is really important since it provides guidelines for a robust dimensioning of the network. Otherwise stated, the planning of an ad hoc network can be made relying on a simplistic deterministic channel model. On the other hand, a factor of great importance is the path loss factor $\alpha$, so that a conservative value of such parameter should be considered in the dimensioning phase. In order to check for the validity of relationship (35), which has been derived under some simplifying approximations, we simulated a $100 \mathrm{~m} \times 100 \mathrm{~m}$, with the same parameters of $\S \mathrm{II}-$ D but with a transmission power of 10 mWatt. Simulations were run for $\sigma=3,4$ and, in order to overcome the border effect, a toroidal distance metric was used [37]. The results, averaged over 1000 simulations, are shown in Fig. 6 in terms of $95 \%$ confidence interval. As it may be seen, the simulation results closely follow the predicted ones for both cases, slightly underestimating the network connection probability.

\section{B. Connectivity for Networks with Unreliable Devices}

The asymptotic analysis can be easily extended to the case of unreliable devices [4], [11], where each device is assumed to be active with probability $p$. We let $p$ be a function of $\lambda$ (which allows for an engineering tradeoff between devices reliability and nodes density), and study scaling laws for the transmission power. Let the channel be characterized by a path

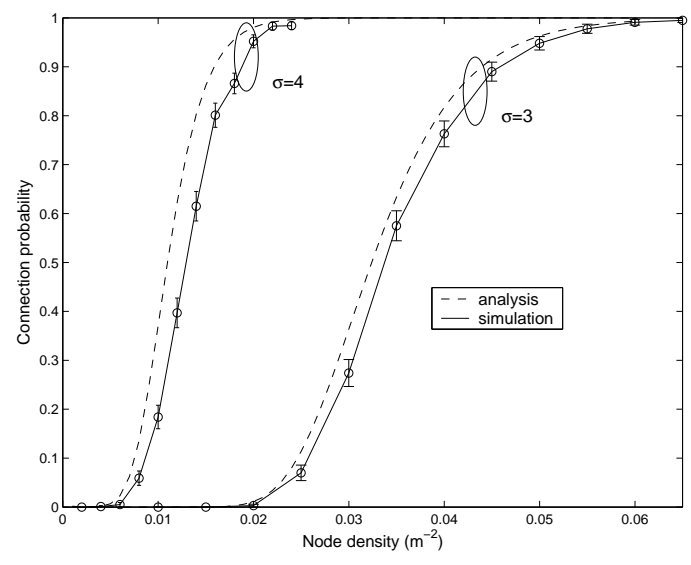

Fig. 6. Connection probability vs. node density in the presence of lognormal shadowing; $P_{t x}=10 \mathrm{mWatt}, K=10, W=0.01 \mathrm{mWatt}, \Psi=10 \mathrm{~dB}$, $\alpha=3.5, \sigma=3,4$.

loss factor $\alpha$, regardless of the possible presence of shadowing and/or Rayleigh fading and let $P_{t x}(\lambda)$ denote the transmission power. Then, under approximations $(i)-($ iii $)$, and threading the footprints of [4], [11], it is easy to show that the followings hold:

(a) a necessary and sufficient condition for asymptotic connectivity is given by:

$$
\lim _{\lambda \rightarrow+\infty} \lambda p(\lambda) e^{-\lambda p(\lambda)\left(P_{t x}(\lambda)\right)^{\frac{2}{\alpha}}}=0 .
$$

(b) If $p=\lim _{\lambda \rightarrow+\infty} p(\lambda)$ satisfies $1<p<0$ and $P_{t x}(\lambda)=$ $\left(\frac{1}{\pi \xi} \cdot \frac{\ln \lambda+c(\lambda)}{\lambda}\right)^{\frac{\alpha}{2}}$, the network is asymptotically connected at distance $d$ if $c(\lambda)$ satisfies

$$
c(\lambda) \geq \zeta \ln \lambda,
$$

where $\zeta>\frac{1-p}{p}$. Furthemore, the network is asymptotically connected only if $c=\lim _{\lambda \rightarrow+\infty} c(\lambda)=+\infty$.

(c) Let $p(\lambda)$ be $p(\lambda)=\lambda^{-\gamma}$, where $0<\gamma<1$. Then a sufficient condition for asymptotic network connectivity at distance $d$ is given by:

$$
p(\lambda)\left[P_{t x}(\lambda)\right]^{\frac{2}{\alpha}} \geq \zeta \frac{\ln \lambda}{\lambda},
$$

where the constant $\zeta$ satisfies $\zeta>1-\gamma$.

\section{IMPROVING NeTWORK CONNECTIVITY THROUGH DIVERSITY}

In this section we explore the possibility of improving the connectivity of the network in the presence of superimposed Rayleigh fading and lognormal shadowing by using multiple antennas at the receiver side. It is clear that, for the time being, the use of multiple antennas for ad hoc networks does not represent a viable solution: the increase in cost, complexity and battery drain for the devices do not allow any practical application of such diversity schemes. However, apart from the theoretical importance of the issue, it is worth noting that diversity can be exploited not only by using multiple antennas, 
but also by means of cooperation schemes [40], [41], where the spatial dimension of the network itself is used as a diversity factor.

Assuming that $M$ antennas are present at the receiver, and that they are spaced apart, so that the paths from the transmitter to the receiver antennas may be considered independent, we study the impact of two signal processing technique, namely:

- best path selection (BPS): among the $M$ received signals, the one with the largest SNR is decoded;

- maximal ratio combining (MRC): the $M$ received signals are combined in such a way to maximize the overall SNR (see [20] and [42] for more details).

It is worth noting that the two models above lead to the same results which could be retrieved by other diversity schemes. For example, the first scheme is equivalent either to a diversity scheme which employs $M$ antennas at the transmitter or to a time diversity scheme, where $M$ replicas of the signal are sent out after a time longer than the coherence time of the channel [43]. On the other hand, performance equivalent to MRC are known to be reachable by employing the wellknown Alamouti scheme [44]. However, in the presence of transmitter-based diversity schemes, a corresponding increase in the transmitted power is required. (In reality, also the adoption of such signal processing techniques implies an additional power consumption.)

Let us focus on the first scheme; given an average SNR of $\Gamma=y$, the probability that at least one path presents a SNR greater than $\Psi$ is:

$$
\begin{aligned}
P_{S \mid \Gamma}(y)=1-\prod_{k=1}^{M} \int_{0}^{\Psi} \mathrm{d} a_{i} f_{\gamma_{i} \mid \Gamma}\left(a_{i} \mid y\right)= \\
=1-\left[\int_{0}^{\Psi} \mathrm{d} a \frac{1}{y} e^{-\frac{a}{y}}\right]^{M}=1-\left(1-e^{-\frac{\Psi}{y}}\right)^{M}= \\
=-\sum_{h=1}^{M}\left(\begin{array}{c}
M \\
h
\end{array}\right)(-1)^{h} e^{-\frac{h \Psi}{y}} .
\end{aligned}
$$

Substituting (42) in (14), together with (15), we get:

$$
\begin{aligned}
P_{I} & =\exp \left\{\lambda \pi \sum_{h=1}^{M}\left(\begin{array}{c}
M \\
h
\end{array}\right)(-1)^{h} \cdot\right. \\
\cdot & {\left[\int_{0}^{+\infty} \mathrm{d} a \int_{0}^{+\infty} \mathrm{d} \rho e^{-\frac{h \Psi W}{a P_{t x}}} 2 \rho \frac{1}{\sqrt{2 \pi} \sigma a} e^{\left.-\frac{1}{2}\left(\frac{\ln \frac{a \rho^{\alpha}}{K}}{\sigma}\right)^{2}\right]}\right]=} \\
& =\exp \left\{\lambda \pi \sum_{h=1}^{M}\left(\begin{array}{c}
M \\
h
\end{array}\right)(-1)^{h} \cdot\right. \\
& \left.\left(\int_{-\infty}^{+\infty} \mathrm{d} x \int_{0}^{+\infty} \mathrm{d} \rho \frac{1}{\sqrt{2 \pi}} e^{-\frac{x^{2}}{2}} 2 \rho e^{-\frac{h \Psi W e^{-\sigma x} \rho^{\alpha}}{K P_{t x}}}\right)\right\}
\end{aligned}
$$

Applying again (28) and (19), we get:

$$
\begin{aligned}
& P_{I}=e^{\left\{\lambda \pi \sum_{h=1}^{M}\left(\begin{array}{c}
M \\
h
\end{array}\right)(-1)^{h} \frac{2}{\alpha} \Gamma\left(\frac{2}{\alpha}\right)\left(\frac{h \Psi W}{K P_{t x}}\right)^{-\frac{2}{\alpha}} e^{\left(\frac{\sqrt{2} \sigma}{\alpha}\right)^{2}}\right\}}= \\
& \left.=e^{\left\{\lambda \pi \frac{2}{\alpha} \Gamma\left(\frac{2}{\alpha}\right)\left(\frac{\Psi W}{K P_{t x}}\right)^{-\frac{2}{\alpha}} e^{\left(\frac{\sqrt{2} \sigma}{\alpha}\right)^{2}} \sum_{h=1}^{M}\left(\begin{array}{c}
M \\
h
\end{array}\right)(-1)^{h} h^{-\frac{2}{\alpha}}\right.}\right\} .
\end{aligned}
$$

Since

$$
\sum_{h=1}^{M}\left(\begin{array}{c}
M \\
h
\end{array}\right)(-1)^{h} h^{-\frac{2}{\alpha}} \leq-1,
$$

we may conclude that the use of receiver diversity with best path selection improves the connectivity properties of the resulting network, as expected. Note, however, that the scaling for asymptotic connectivity remains unchanged.

In case of MRC, the system behaves as if the channel consisted of an $M$-Nakagami fading superimposed to lognormal shadowing [20]. The pdf of the instantaneous SNR is given by:

$$
f_{\gamma \mid \Gamma}(a \mid y)=\left(\frac{M}{y}\right)^{M} \frac{a^{M-1}}{\Gamma(M)} e^{-M \frac{a}{y}},
$$

where $\Gamma(\cdot)$ is the Gamma function. Note that for $M=1$ we obtain the usual expression for Rayleigh fading. The ccdf is given by:

$$
P_{S \mid \Gamma}(y)=P[\gamma>\Psi \mid \Gamma=y]=1-F_{\gamma}(\Psi)=\frac{\Gamma\left(M, \frac{M \Psi}{y}\right)}{\Gamma(M)},
$$

where $\Gamma(\cdot, \cdot)$ is the incomplete Gamma function [29]. Since $M$ is an integer, the expression above simplifies to:

$$
P_{S \mid \Gamma}(y)=e^{-M \frac{\Psi}{y}} \sum_{h=0}^{M-1}\left(\frac{M \Psi}{y}\right)^{h} \frac{1}{(h+1) !} .
$$

Proceeding as above, the node isolation probability turns out to be:

$$
\begin{gathered}
P_{I}=\exp \left\{-\lambda \pi \sum_{h=0}^{M-1} \frac{1}{(h+1) !}\left[\int_{0}^{+\infty} \mathrm{d} a \int_{0}^{+\infty} \mathrm{d} \rho e^{-\frac{\Psi W}{a P_{t x}}}\right.\right. \\
\left.\left.\cdot\left(\frac{M \Psi W}{a P_{t x}}\right)^{h} 2 \rho \frac{1}{\sqrt{2 \pi} \sigma a} e^{-\frac{1}{2}\left(\frac{\ln a-\ln \left(K \rho^{-\alpha}\right)}{\sigma}\right)^{2}}\right]\right\}
\end{gathered}
$$

that after some algebra reduces to:

$$
\begin{aligned}
P_{I}=\exp \left\{-\lambda \pi \frac{2}{\alpha} e^{\left(\frac{\sqrt{2} \sigma}{\alpha}\right)^{2}}\left(\frac{\Psi W}{K P_{t x}}\right)^{-\frac{2}{\alpha}} .\right. \\
\left.\cdot \sum_{h=0}^{M-1} \frac{1}{(h+1) !} M^{h} \Gamma\left(h+\frac{2}{\alpha}\right)\right\} .
\end{aligned}
$$

Since

$$
\sum_{h=0}^{M-1} \frac{1}{(h+1) !} M^{h} \Gamma\left(h+\frac{2}{\alpha}\right) \geq \Gamma\left(\frac{2}{\alpha}\right)
$$


also receiver-based diversity with MRC improves the network connectivity.

For both BPS and MRC, the connectivity improvement could be predicted by estabilishing a stochastic ordering among the distributions of the communication range $R$ in the presence of different number of antennas. It suffices to note that for both schemes, if $M_{1} \leq M_{2}, P_{S \mid \Gamma, M_{1}} \leq P_{S \mid \Gamma, M_{2}}$, where $P_{S \mid \Gamma, x}$ is the probability of successful reception given an average SNR of $\Gamma$ and in the presence of $x$ antennas. Substituting in (6), it is easy to see that a stochastic ordering among the distribution corresponding to different values of $M$ is possible:

$$
R_{M_{1}} \preceq R_{M_{2}} \quad M_{1} \leq M_{2},
$$

and the connectivity improvement follows along the arguments outlined in $\S$ II-D.

Some results, in terms of coverage probability, for both the schemes analysed, are reported in Fig. 7 and Fig. 8. As it may be seen, both schemes improves network performance. It is worth noting that with BPS the coverage enhancements tend to saturate, so that it does not seem of interest to go beyond $M=3$, while with MRC the performance improvement tends to considerably increase with $M$. A direct comparison of the performance achieved by both schemes is represented in Fig. 9, where it may be seen that MRC outperforms the simpler BPS scheme, and that the performance achievable with BPS and $M=4$ antennas may be obtained also by limiting the number of antennas to $M=2$ and employing a MRC scheme.

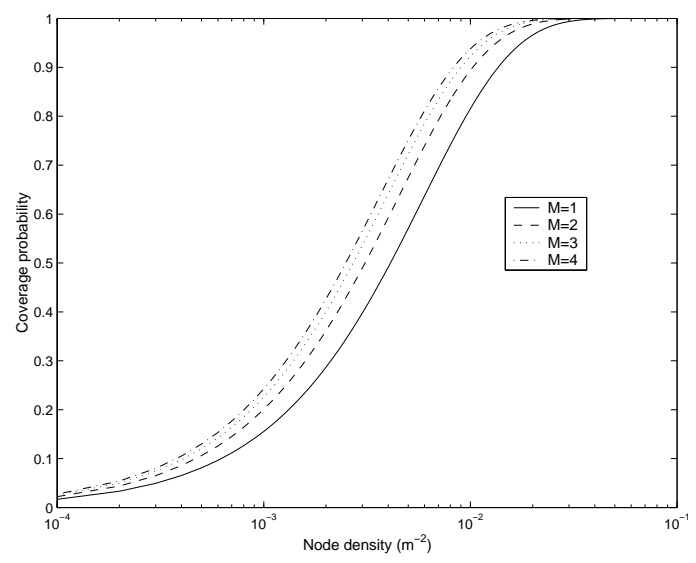

Fig. 7. Coverage probability vs. node density: the impact of receiver diversity with best path selection algorithm; $P_{t x}=1$ mWatt, $K=10, W=0.01$ mWatt, $\Psi=10 \mathrm{~dB}, \alpha=3.5, \sigma=3$

\section{CONCLUSIONS}

In this paper we have presented an analytical procedure for the computation of the node isolation probability in an ad hoc network in the presence of channel randomness, with a particular emphasis on the effect of lognormal shadowing and Rayleigh fading phenomena. We have shown that the node isolation probability decreases as the lognormal spread $\sigma$ increases, and investigated the negative effect of Rayleigh fading. We proved that no connectivity improvement may be achieved by using random transmission power selection

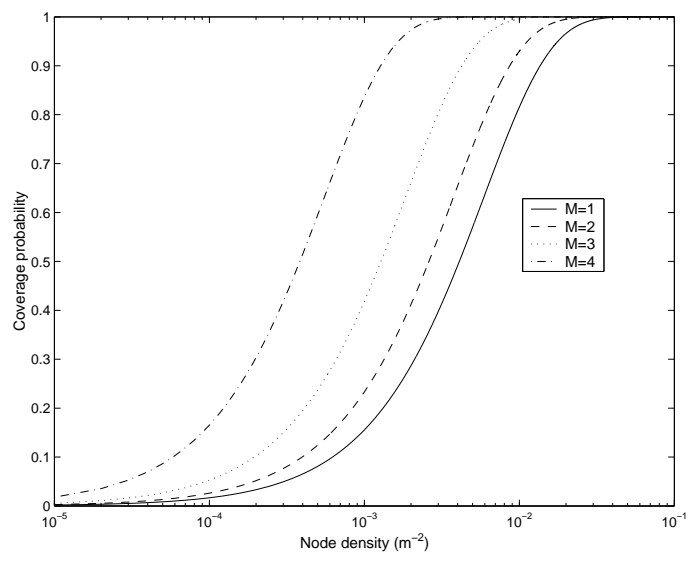

Fig. 8. Coverage probability vs. node density: the impact of receiver diversity with maximal ratio combining; $P_{t x}=1 \mathrm{mWatt}, K=10, W=0.01 \mathrm{mWatt}$, $\Psi=10 \mathrm{~dB}, \alpha=3.5, \sigma=3$

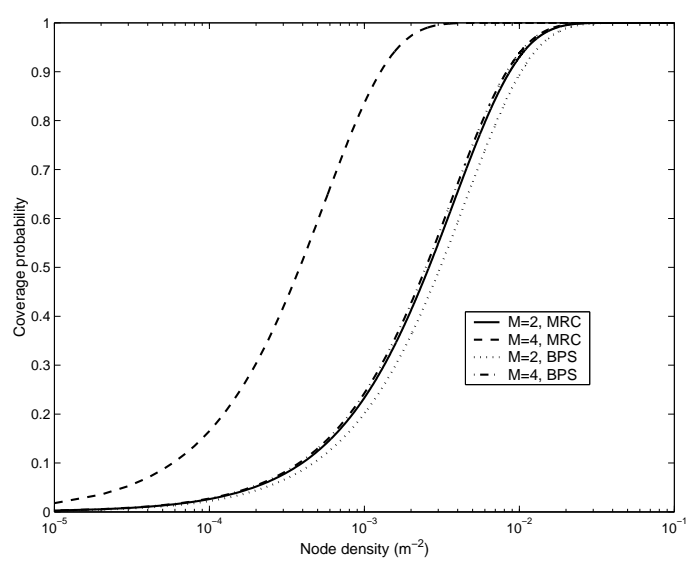

Fig. 9. Coverage probability vs. node density: comparison of best path selection (BPS) and maximal ratio combining (MRC); $P_{t x}=1 \mathrm{mWatt}, K=$ $10, W=0.01$ mWatt, $\Psi=10 \mathrm{~dB}, \alpha=3.5, \sigma=3$

schemes. The node isolation probability coincides with the complement of the coverage probability, given that nodes are distributed according to a Poisson point process; otherwise it is possible to relate the two quantities through an inversion formula.

From the node isolation probability we can get, under some simplifying approximations, a closed form formula for the probability of the network being connected in the presence of a very large device density. Simulation results have been presented, which show the soundness of the proposed procedure.

For the case of superimposed shadowing and Rayleigh fading, the connectivity improvement achievable by means of two simple diversity schemes, best path selection and maximal ratio combining, has been investigated; a general stochastic ordering relationship has been found for both schemes, showing the beneficial impact of the use of diversity schemes.

Directions for future work include the generalization of the results to more general node placement distributions and a study of the impact of various mobility patterns on the 
connectivity results found for a static scenario.

\section{ACKNOWLEDGMENTS}

This work was supported by the EURO NGI Network of Excellence. This work has been done while D. Miorandi was visiting the MAESTRO project at INRIA Sophia Antipolis. The authors would like to thank R. Verdone and J. Orriss for the useful discussions on the subject.

\section{REFERENCES}

[1] I. F. Akyildiz, W. Su, Y. Sankarasubramaniam, and E. Cayirci, "Wireless sensor networks: A survey," Computer Networks, vol. 38, no. 4, pp. 393422, Mar. 2002.

[2] P. Gupta and P. R. Kumar, "Critical power for asymptotic connectivity," in Proc. of IEEE CDC, Tampa, USA, 1998.

[3] — "The capacity of wireless networks," IEEE Trans. on Inf. Th. vol. 46, no. 2, pp. 388-404, Mar. 2000.

[4] S. Shakkottai, R. Srikant, and N. B. Shroff, "Unreliable sensor grids: coverage, connectivity and diameter," in Proc. of IEEE INFOCOM, San Francisco, CA, 2003.

[5] P. Santi and D. M. Blough, "The critical transmitting range for connectivity in sparse wireless ad hoc networks," IEEE Trans. on Mob. Comp., vol. 2, no. 1, pp. 25-39, Jan-Mar 2003.

[6] O. Dousse, P. Thiran, and M. Hasler, "Connectivity in ad-hoc and hybrid networks," in Proc. of IEEE INFOCOM, New York, USA, 2002.

[7] A. Rajeswaran and R. Negi, "Capacity of power constrained ad-hoc networks," in Proc. of IEEE INFOCOM, Hong Kong, 2004.

[8] L. Booth, J. Bruck, M. Cook, and M. Franceschetti, "Ad hoc wireless networks with noisy links," in Proc. of IEEE ISIT '03, Yokohama, Japan, 2003.

[9] C. Bettstetter and C. Hartmann, "Connectivity of wireless multihop networks in a shadow fading environment," in Proc. of ACM MSWiM, San Diego, CA, 2003.

[10] R. Hekmat and P. Van Mieghem, "Study of connectivity in wireless ad-hoc networks with an improved radio model," in Proc. of WiOpt, Cambridge, UK, 2004.

[11] D. Miorandi and E. Altman, "Connectivity in ad-hoc networks: a queueing theoretical approach," in Proc. of WiOpt, Cambridge, UK, 2004.

[12] M. Zorzi and S. Pupolin, "Outage probability in multiple access packet radio networks in the presence of fading," IEEE Trans. Veh. Tech., vol. 43, no. 3, pp. 604-610, Aug 1994.

[13] J. Orriss and S. K. Barton, "Probability distributions for the number of radio transceivers which can communicate with one another," IEEE Trans. Comm., vol. 51, no. 4, pp. 676-681, Apr. 2003.

[14] _ - "A statistical model for connectivity between mobiles and base stations: the extension to Suzuki," 2002, COST 273 TD(02) 121.

[15] — "A statistical model for connectivity between mobiles and base stations: from Suzuki to Rice and beyond," 2003, COST $273 \operatorname{TD}(03)$ 89.

[16] O. Dousse, F. Baccelli, and P. Thiran, "Impact of interferences on connectivity in ad-hoc networks," in Proc. of IEEE INFOCOM, San Francisco, USA, 2003.

[17] F. Baccelli and B. Blaszczyszyn, "On a coverage process ranging from the Boolean model to the Poisson Voronoi tessellation with applications to wireless communications," INRIA, Tech. Rep. 4019, Oct. 2000.

[18] O. Dousse and P. Thiran, "Connectivity vs capacity in dense ad hoc networks," in Proc. of IEEE INFOCOM, Hong Kong, 2004.

[19] T. S. Rappaport, Wireless Communications. Upper Saddle River, New Jersey: Prentice Hall, 1999.

[20] L. Hanzo, C. H. Wong, and M. S. Yee, Adaptive Wireless Transceivers. New York: John Wiley and Sons, 2002.

[21] D. Stoyan, W. S. Kendall, and J. Mecke, Stochastic geometry and its applications. New York: J. Wiley \& Sons, 1987.

[22] P. Hall, Introduction to the theory of coverage processes. New York: J. Wiley and sons, 1988

[23] S. Meguerdichian, F. Koushanfar, M. Potkonjak, and M. B. Srivastava, "Coverage problems in wireless ad-hoc sensor networks," in Proc. of IEEE INFOCOM, Anchorage, 2001.
[24] B. Liu and D. Towsley, "On the coverage and detectability of large-scale wireless sensor networks," in Proc. of WiOpt, Sophia Antipolis, France, 2003.

[25] K. Kar and S. Banerjee, "Node placement for connected coverage in sensor networks," in Proc. of WiOpt, Sophia Antipolis, France, 2003.

[26] X. Wang, G. Xing, Y. Zhang, C. Lu, R. Pless, and C. Gill, "Integrated coverage and connectivity configuration in wireless sensor networks," in Proc. of SenSys, Los Angeles, CA, 2003.

[27] X.-Y. Li, P.-J. Wan, and O. Frieder, "Coverage in wireless ad hoc sensor networks," IEEE Trans. on Comp., vol. 52, no. 6, pp. 753-763, Jun 2003.

[28] R. Meester and R. Roy, Continuum Percolation. New York: Cambridge Univ. Press, 1996.

[29] I. S. Gradshteyn and I. M. Ryzhik, Table of integrals, series, and products. Orlando: Academic Press, 1983.

[30] D. Stoyan, Comparison Methods for Queues and Other Stochasti Models. New York: John Wiley \& Sons, 1983.

[31] B. Bollobás, Random graphs. Cambridge, UK: Cambridge University Press, 2001.

[32] M. D. Penrose, Random geometric graphs. Oxford, UK: Oxford University Press, 2003.

[33] C. Bettstetter, "On the minimum node degree and connectivity of a wireless multihop network," in Proc. of Mobihoc, Lausanne, CH, 2002.

[34] R. Albert and A. L. Barabási, "Statistical mechanics of complex networks," Reviews of Modern Physics, vol. 74, pp. 47-97, Jan. 2002.

[35] A. Helmy, "Small worlds in wireless networks," IEEE Communications Letters, no. 10, pp. 490-492, Oct. 2003.

[36] M. Franceschetti, L. Booth, J. Bruck, M.Cook, and R. Meester, "Continuum percolation with unreliable and spread out connections," 2004, journal of Statistical Physics. In press. [Online]. Available: http://fleece.ucsd.edu/ massimo/Journal/PercSpread-Submission.pdf

[37] C. Bettstetter and O. Krause, "On border effects in modeling and simulation of wireless ad hoc networks," in Proc. of IEEE MWCN, Recife, Brazil, 2001.

[38] M. D. Penrose, "The longest edge of the random minimal spanning tree," The Annals of Appl. Prob., vol. 7, no. 2, pp. 340-361, 1997.

[39] P. Hall, "Heavy traffic approximations for busy period in an $M|G| \infty$ queue," Stochastic Processes and their Applications, vol. 19, pp. 259269, 1985.

[40] L.-L. Xie and P. R. Kumar, "A network information theory for wireless communications: scaling laws and optimal operation," IEEE Trans. Inf. Th., vol. 50, no. 5, pp. 748-767, May 2004.

[41] J. N. Laneman, D. N. C. Tse, and G. W. Wornell, "Cooperative diversity in wireless networks: efficient protocol and outage behavior," IEEE Trans. Inf. Th., 2004, to appear. [Online]. Available: http://www.nd.edu/ jnl/pubs/it2002.pdf

[42] N. Benevenuto and G. Cherubini, Algorithms for Communications Systems and Their Applications. New York: John Wiley \& Sons, 2002.

[43] J. H. Winters, "The diversity gain of transmit diversity in wireles systems with Rayleigh fading," in Proc. of ICC, New York, NY, 1994.

[44] S. M. Alamouti, "A simple transmit diversity technique for wireless communications," IEEE J. Sel. Areas on Comm., vol. 16, no. 8, pp. 1451-1458, Oct. 1998

\section{APPENDIX}

In order to carry out the computation of the node isolation probability, we may follow an approach inspired by that of Zorzi et al. [12] for the analysis of the outage probability in packet radio networks. We consider a ball of radius $c$, $D_{c}$ and condition on the number of nodes present, denoted by $k$. Notice that the $k$ points of $\mathcal{N}$ in $D_{c}$ are uniformly distributed. We compute the node isolation probability under such conditions. Then, we apply the total probability theorem to uncondition on $k$ (noting that the number of nodes present in $D_{c}$ forms a Poisson random variable of intensity $\lambda \pi c^{2}$ ). Finally, we pass to the limit $c \rightarrow+\infty$ to get the node isolation 
probability. Thus:

$$
\begin{gathered}
P_{I}=\lim _{c \rightarrow+\infty} \sum_{k=0}^{\infty} e^{-\lambda \pi c^{2}} \frac{\left(\lambda \pi c^{2}\right)^{k}}{k !} \int_{0}^{+\infty} \mathrm{d} a_{1} \int_{0}^{+\infty} \mathrm{d} a_{k} . \\
\prod_{i=1}^{k}\left(1-P_{S \mid \Gamma}\left(\frac{a_{i} P_{t x}}{W}\right)\right) \prod_{i=0}^{k} \int_{0}^{c} \frac{2 \rho_{i} \mathrm{~d} \rho_{i}}{c^{2}} f_{l \mid r}\left(a_{i} \mid \rho_{i}\right) .
\end{gathered}
$$

Then,

$$
\begin{aligned}
& P_{I}=\lim _{c \rightarrow+\infty} \sum_{k=0}^{\infty} e^{-\lambda \pi c^{2}} \frac{\left(\lambda \pi c^{2}\right)^{k}}{k !} \prod_{i=1}^{k} \int_{0}^{+\infty} \mathrm{d} a_{i} . \\
& \cdot\left(1-P_{S \mid \Gamma}\left(\frac{a_{i} P_{t x}}{W}\right)\right) \int_{0}^{c} \frac{2 \rho_{i} \mathrm{~d} \rho_{i}}{c^{2}} f_{l \mid r}\left(a_{i} \mid \rho_{i}\right)= \\
& =\lim _{c \rightarrow+\infty} \sum_{k=0}^{\infty} e^{-\lambda \pi c^{2}} \frac{\left(\lambda \pi c^{2}\right)^{k}}{k !}\left[\int_{0}^{+\infty} \mathrm{d} a .\right. \\
& \left.\cdot\left(1-P_{S \mid \Gamma}\left(\frac{a P_{t x}}{W}\right)\right) \int_{0}^{c} \frac{2 \rho \mathrm{d} \rho}{c^{2}} f_{l \mid r}(a \mid \rho)\right]^{k}= \\
& =\lim _{c \rightarrow+\infty} e^{-\lambda \pi c^{2}\left[1-\int_{0}^{+\infty} \mathrm{d} a \int_{0}^{c} \mathrm{~d} \rho\left(1-P_{S \mid \Gamma}\left(\frac{a P_{t x}}{W}\right)\right) \frac{2 \rho}{c^{2}} f_{l \mid r}(a \mid \rho)\right]}= \\
& =e^{-\lambda \pi \lim _{c \rightarrow+\infty}\left[c^{2}-\int_{0}^{+\infty} \mathrm{d} a \int_{0}^{c} \mathrm{~d} \rho\left(1-P_{S \mid \Gamma}\left(\frac{a P_{t x}}{W}\right)\right) 2 \rho f_{l \mid r}(a \mid \rho)\right]}= \\
& =e^{-\lambda \pi \lim _{c \rightarrow+\infty}\left[\int_{0}^{+\infty} \mathrm{d} a \int_{0}^{c} \mathrm{~d} \rho P_{S \mid \Gamma}\left(\frac{a P_{t x}}{W}\right) 2 \rho f_{l \mid r}(a \mid \rho)\right]}= \\
& =e^{-\lambda \pi\left[\int_{0}^{+\infty} \mathrm{d} a \int_{0}^{+\infty} \mathrm{d} \rho P_{S \mid \Gamma}\left(\frac{a P_{t x}}{W}\right) 2 \rho f_{l \mid r}(a \mid \rho)\right]}
\end{aligned}
$$

where the passage to the limit is justified if the integral converges absolutely (this corresponds to the condition $E\left[R^{2}\right]<$ $+\infty)$.

Substituting (6) in (51) we retrieve (14). 